PROCEEDINGS OF THE

AMERICAN MATHEMATICAL SOCIETY

Volume 125, Number 4, April 1997, Pages 1081-1087

S 0002-9939(97)03660-5

\title{
ON QUASISPECTRAL MAXIMAL SUBSPACES OF A CLASS OF VOLTERRA-TYPE OPERATORS
}

\author{
ROMAN DRNOVŠEK
}

(Communicated by Palle E. T. Jorgensen)

\begin{abstract}
The concept of quasispectral maximal subspaces for quasinilpotent (but not nilpotent) operators was introduced by M. Omladič in 1984. As an application a class of quasinilpotent operators on $L^{p}$-spaces, close to the Volterra kernel operator, was studied. In the present Banach function space setting we determine all quasispectral maximal subspaces of analogues of such operators and prove that these subspaces are all the invariant bands. An example is given showing that (in general) they are not all the closed, invariant ideals of the operator.
\end{abstract}

\section{INTRODUCTION}

In the paper [1] the following notions of local quasispectral radius and quasispectral maximal subspaces are introduced. Denote by $\mathcal{B}(X)$ the algebra of all bounded operators acting on a non-trivial complex Banach space $X$. Suppose that $T \in \mathcal{B}(X)$ is not nilpotent. For any $x \in X$, the local quasispectral radius is defined by

$$
r_{T}(x)=\limsup _{n \rightarrow \infty}\left(\frac{\left\|T^{n} x\right\|}{\left\|T^{n}\right\|}\right)^{1 / n} .
$$

Let $Y$ be a closed subspace of $X$, invariant under $T$. We denote by $\left.T^{n}\right|_{Y}$ the restriction of $T^{n}$ to $Y$. Define

$$
r_{T}(Y)=\limsup _{n \rightarrow \infty}\left(\frac{\left\|\left.T^{n}\right|_{Y}\right\|}{\left\|T^{n}\right\|}\right)^{1 / n} .
$$

It is proved in $[1$, proposition 2.3$]$ that

$$
r_{T}(Y)=\sup \left\{r_{T}(y): y \in Y\right\} .
$$

A closed subspace $Y$ of $X$, invariant under $T$, is said to be a quasispectral maximal subspace of $T$ if for every closed subspace $Z$ of $X$, invariant under $T$ as well, we have $Z \subseteq Y$ whenever $r_{T}(Z) \leq r_{T}(Y)$.

For any $a \in[0,1]$, define

$$
X_{T}(a)=\left\{x \in X: r_{T}(x) \leq a\right\} .
$$

Received by the editors July 6, 1995 and, in revised form, September 22, 1995.

1991 Mathematics Subject Classification. Primary 47B38, 47A15.

Key words and phrases. Banach function spaces, operators, invariant subspaces.

This work was supported in part by the Research Ministry of Slovenia.

(C)1997 American Mathematical Society 
It is shown in [1, theorem 3.1] that $X_{T}(a)$ is a quasispectral maximal subspace of $T$ whenever it is closed.

In [1] the definition of quasispectral maximal subspaces was applied to a class of quasinilpotent operators on $L^{p}$-spaces, close to the Volterra kernel operator. We reduce the number of assumptions, improve the proof of the main result (theorem 4.4) in [1], and extend the theorem to Banach function spaces. If such an operator is injective, we determine all its quasispectral maximal subspaces. We also prove that these subspaces are precisely the family of all invariant bands. An example shows that in general they are not all the invariant, closed ideals. For the theory of Banach function spaces and Banach lattices we refer the reader to [4] (for the theory of Banach lattices only, see also [3]). We will recall some of the relevant facts.

Let $\mu$ be a $\sigma$-finite positive measure on a $\sigma$-algebra $\Sigma$ of subsets of a non-void set $E$. Let $M(E, \mu)$ be the vector space of all equivalence classes of complex $\Sigma$ measurable functions on $E$. A Banach lattice $L$ is called a Banach function space if it is an ideal of $M(E, \mu)$, i.e., $f \in L, g \in M(E, \mu)$, and $|g| \leq|f|$ imply that $g \in L$. An ideal $I$ of a Banach function space $L$ is a band if for any subset $A \subset I$ of real functions with $\sup A \in L$ we have $\sup A \in I$. Recall that bands are necessarily closed subspaces.

We will always assume that $E$ is the carrier of $L$, that is, there is no subset $F$ of $E$ of positive measure with the property that $f=0$ a.e. on $F$ for all $f \in L$ (see [4]). By $L^{\prime}$ we denote the associate space (also called the Köthe dual) of all $g \in M(E, \mu)$ such that

$$
\phi(f)=\int_{E} f(t) g(t) d \mu(t)
$$

defines a bounded linear functional $\phi$ on $L$. The space $L^{\prime}$ is also a Banach function space with respect to the associate norm $\|\cdot\|^{\prime}$ defined by

$$
\|g\|^{\prime}=\sup \left\{\int_{E}|f(t) g(t)| d \mu(t):\|f\| \leq 1\right\},
$$

and it may be considered as a closed subspace of the dual Banach lattice $L^{*}$. Clearly, we have

$$
\int_{E}|f(t) g(t)| d \mu(t) \leq\|f\|\|g\|^{\prime}
$$

for every $f \in L$ and every $g \in L^{\prime}$.

We proceed to define the operator that is studied in this paper. Let $\phi: E \rightarrow[0,1]$ be a function such that the set $E_{t}=\{s \in E: \phi(s) \leq \phi(t)\}$ is measurable for every $t \in E$. It is easy to verify that the function $\phi$ is measurable. We assume further that

$$
\mu(\{s \in E: \phi(s)=\phi(t)\})=0
$$

for every $t \in E$.

Let $u \in L$ and $v \in L^{\prime}$ be strictly positive functions. Note that such functions always exist. Indeed, by [4, theorem 86.2] there exists a sequence $E_{n} \uparrow E$ such that $0<\mu\left(E_{n}\right)<\infty$ and $\chi_{E_{n}} \in L$ for all $n$. It follows that the function $u$ defined by

$$
u=\sum_{n=1}^{\infty} \frac{1}{2^{n}\left\|\chi_{E_{n}}\right\|} \chi_{E_{n}}
$$


belongs to $L$ and is strictly positive, i.e., $u(t)>0$ almost everywhere on $E$.

Define the operator $V$ on the Banach function space $L$ by

$$
(V f)(t)=u(t) \int_{E_{t}} f(s) v(s) d \mu(s)=u(t) \int_{\phi(s)<\phi(t)} f(s) v(s) d \mu(s),
$$

for every $f \in L$ and for almost all $t \in E$. Since $|V f|(t) \leq u(t)\|f\|\left\|v \chi_{E_{t}}\right\|^{\prime} \leq$ $u(t)\|f\|\|v\|^{\prime}$, we conclude that $\|V f\| \leq\|f\|\|v\|^{\prime}\|u\|$ for all $f \in L$, and hence $\|V\| \leq\|v\|^{\prime}\|u\|$. Therefore, the operator $V$ is well-defined and bounded.

We conclude this section by showing that we can restrict our attention to the case when $\mu(E)=1$ and $u=v=e$, where $e$ denotes the measurable function identically equal to 1 . First, since the number $c=\int_{E} u v d \mu$ belongs to $(0, \infty)$ and since we shall consider only invariant subspaces of $V$, we replace $u$ by $(1 / c) u$, so that $\int_{E} u v d \mu=1$. Consequently, if $m$ is the measure on $\Sigma$ defined by $m(A)=\int_{A} u v d \mu$, then $m(E)=1$. It is also clear that $m$ is equivalent with $\mu$, i.e., $m(A)=0$ if and only if $\mu(A)=0$. Hence $M(E, m)=M(E, \mu)$. Let us now define a Banach function space $L_{m} \subseteq M(E, m)$ so that the map $L \rightarrow L_{m}$ defined by $f \rightarrow f / u$ is an isometric isomorphism of Banach lattices. In view of this isomorphism the operator $V_{m}$ on $L_{m}$ defined by

$$
\left(V_{m} f\right)(t)=\int_{E_{t}} f(s) d m(s),
$$

for every $f \in L_{m}$ and for almost all $t \in E$, corresponds to $V$. Let $L_{m}^{\prime}$ be the associate space of $L_{m}$ with respect to the measure $m$. It is easy to check that the map $L^{\prime} \rightarrow L_{m}^{\prime}$ defined by $g \rightarrow g / v$ is an isometric isomorphism of Banach lattices. Evidently, $e \in L_{m} \cap L_{m}^{\prime}$, i.e., $L^{\infty}(E, m)$ is a subspace of both $L_{m}$ and $L_{m}^{\prime}$. For the remainder of this paper we can therefore assume that $\mu(E)=1$ and $u=v=e$.

\section{Auxiliary Results}

For each $t \in E$ define $\psi(t)=\mu\left(E_{t}\right)$ and $F_{t}=\{s \in E: \psi(s) \leq \psi(t)\}$. We assume from now on that $\left\{F_{t}: t \in E\right\} \subseteq \Sigma$. It is clear from the proof of the next proposition that this hypothesis is satisfied if the measure $\mu$ is complete.

Proposition 2.1. For each $t \in E$ the set $F_{t}$ contains $E_{t}$ and $\mu\left(F_{t}-E_{t}\right)=0$. In particular, $\mu\left(F_{t}\right)=\psi(t)$. Furthermore, the function $\psi$ is measurable and

$$
\mu(\{s \in E: \psi(s)=\psi(t)\})=0
$$

for every $t \in E$.

Proof. Since $\phi(s) \leq \phi(t)$ implies $\psi(s) \leq \psi(t)$, we have $E_{t} \subseteq F_{t}$. Fix $t \in E$. Set $a=\sup \{\phi(s): \psi(s)=\psi(t)\}$, and choose a sequence $\left\{s_{n}\right\}$ in $E$ such that the sequence $\left\{\phi\left(s_{n}\right)\right\}$ increases to $a$ and such that $\phi\left(s_{n}\right) \geq \phi(t)$ for all $n$. (If $\phi(s)=a$ for some $s \in E$, then define $s_{n}=s$.) It is clear that $E_{t} \subseteq E_{s_{n}}$. Also, $\psi(t)=\psi\left(s_{n}\right)$ by the definition of $a$, so that $\mu\left(E_{t}\right)=\mu\left(E_{s_{n}}\right)$. Hence, $\mu\left(E_{s_{n}}-E_{t}\right)=0$, and so $\mu\left(\bigcup_{n=1}^{\infty} E_{s_{n}}-E_{t}\right)=0$. For the proof of the first assertion it is therefore enough to show that $F_{t} \subseteq \bigcup_{n=1}^{\infty} E_{s_{n}}$. To this end, choose $u \notin \bigcup_{n=1}^{\infty} E_{s_{n}}$. Then $\phi(u)>\phi\left(s_{n}\right)$ for all $n$. By the definition of $a$ we then have $\psi(u)>\psi(t)$, so that $u \notin F_{t}$.

The proof of the measurability of the function $\psi$ is the same as that of $\phi$. For the proof of (2), we observe first that we have already proved that $\mu(\{s \in E: \psi(s)=$ $\psi(t), \phi(s)>\phi(t)\})=0$. Set $b=\inf \{\phi(s): \psi(s)=\psi(t)\}$. Similarly as above one can show that $\mu(\{s \in E: \psi(s)=\psi(t), b<\phi(s) \leq \phi(t)\})=0$. By (1) we finally obtain (2). 
In view of Proposition 2.1 we may (and do) assume from now on that $\mu\left(E_{t}\right)=$ $\phi(t)$ for all $t \in E$, replacing $E_{t}$ and $\phi$ by $F_{t}$ and $\psi$ respectively, if necessary.

It is not difficult to show that $\sup \{\phi(t): t \in E\}=1$ and $\inf \{\phi(t): t \in E\}=0$. Moreover, we have the following

Proposition 2.2. If $0 \leq a \leq b \leq 1$, then $\mu(\{t \in E: a \leq \phi(t) \leq b\})=b-a$.

Proof. It is easy to see that we have to prove only the case when $a=0$ and $0<b<1$. Setting $c=\sup \{\phi(t): \phi(t) \leq b\}$ and $d=\inf \{\phi(t): \phi(t) \geq b\}$, we choose sequences $\left\{s_{n}\right\}$ and $\left\{t_{n}\right\}$ in $E$ with $\phi\left(s_{n}\right) \uparrow c$ and $\phi\left(t_{n}\right) \downarrow d$. Then

$$
\begin{gathered}
\phi\left(s_{n}\right)=\mu\left(E_{s_{n}}\right) \rightarrow \mu(\{t \in E: \phi(t) \leq c\})=\mu(\{t \in E: \phi(t) \leq b\}) \text { and } \\
\phi\left(t_{n}\right)=\mu\left(E_{t_{n}}\right) \rightarrow \mu(\{t \in E: \phi(t) \leq d\})=\mu(\{t \in E: \phi(t) \leq b\}),
\end{gathered}
$$

where we have used (1). Thus, $c=d=b$ and $\mu(\{t \in E: \phi(t) \leq b\})=b$.

Proposition 2.3. Let $a \in[0,1], n \in \mathbb{N}$, and let $A=\{s \in E: \phi(s) \geq a\}$. Then

$$
V^{n} \chi_{A}=\frac{(\phi-a)^{n} \chi_{A}}{n !} \text {. }
$$

Proof. For almost all $t \in E$ we have

$$
\begin{aligned}
& \left(V^{n} \chi_{A}\right)(t)=\int_{a<\phi\left(t_{1}\right)<\phi(t)} d \mu\left(t_{1}\right) \int_{a<\phi\left(t_{2}\right)<\phi\left(t_{1}\right)} d \mu\left(t_{2}\right) \cdot \ldots \cdot \int_{a<\phi\left(t_{n}\right)<\phi\left(t_{n-1}\right)} d \mu\left(t_{n}\right) \\
& \quad=\int_{a<\phi\left(t_{n}\right)<\phi\left(t_{n-1}\right)<\ldots<\phi\left(t_{1}\right)<\phi(t)} d \mu\left(t_{1}\right) \ldots d \mu\left(t_{n}\right) .
\end{aligned}
$$

The result is clear if $\phi(t)<a$, so we assume that $\phi(t) \geq a$. Let $\sigma$ be any permutation of the set $\{1,2,3, \ldots, n\}$. Clearly, the integral

$$
\int_{a<\phi\left(t_{\sigma(n)}\right)<\phi\left(t_{\sigma(n-1)}\right)<\ldots<\phi\left(t_{\sigma(1)}\right)<\phi(t)} d \mu\left(t_{1}\right) \ldots d \mu\left(t_{n}\right)
$$

does not depend on the choice of $\sigma$, and the sum of such integrals over all permutations is equal to

$$
\left(\int_{a<\phi(s)<\phi(t)} d \mu(s)\right)^{n}=(\mu(\{s \in E: a<\phi(s)<\phi(t)\}))^{n}=(\phi(t)-a)^{n},
$$

where we have used (1) and Proposition 2.2. This implies the assertion.

Proposition 2.4. If $a \in[0,1]$ and $A=\{s \in E: \phi(s) \geq a\}$, then

$$
\lim _{n \rightarrow \infty}\left\|(\phi-a)^{n} \chi_{A}\right\|^{1 / n}=1-a .
$$

Proof. Since the result is clear when $a=1$, we assume $a<1$. For any $c \in[a, 1)$ the measure of the set $C=\{s \in E: \phi(s) \geq c\}$ is positive by Proposition 2.2. Since $(c-a) \cdot \chi_{C} \leq(\phi-a) \cdot \chi_{A} \leq(1-a) \cdot e$, we obtain

$$
c-a \leq \liminf _{n \rightarrow \infty}\left\|(\phi-a)^{n} \chi_{A}\right\|^{1 / n} \leq \limsup _{n \rightarrow \infty}\left\|(\phi-a)^{n} \chi_{A}\right\|^{1 / n} \leq 1-a,
$$

which establishes the formula. 
Proposition 2.5. If $n \in \mathbb{N}$ and $f \in L$, then

$$
(n-1) !\left(V^{n} f\right)(t)=\int_{\phi(s)<\phi(t)}(\phi(t)-\phi(s))^{n-1} f(s) d \mu(s)
$$

for almost all $t \in E$.

Proof. We may assume that $f$ is a nonnegative function. Put $A(s)=\{u \in E$ : $\phi(u) \geq \phi(s)\}$ for each $s \in E$. An application of Fubini's theorem gives the equality

$$
\left(V^{n} f\right)(t)=\int_{\phi(s)<\phi(t)} f(s) d \mu(s) \int_{\phi(s)<\phi\left(t_{n-1}\right)<\ldots<\phi\left(t_{1}\right)<\phi(t)} d \mu\left(t_{1}\right) \ldots d \mu\left(t_{n-1}\right),
$$

which holds almost everywhere on $E$. By (3) it follows that

$$
\left(V^{n} f\right)(t)=\int_{\phi(s)<\phi(t)} f(s)\left(V^{n-1}\left(\chi_{A(s)}\right)\right)(t) d \mu(s)
$$

for almost all $t \in E$. Now apply Proposition 2.3 to obtain the desired equality.

For $a \in[0,1]$, denote by $B(a)$ the set of all $f \in L$ for which $f(t)=0$ for almost all $t \in E$ with $\phi(t) \leq a$. Note that $B(a)$ is a band in $L$, invariant under $V$.

Proposition 2.6. Let $a \in[0,1], n \in \mathbb{N}$, and let $f \in B(a)$. Then

$$
(n-1) !\left\|V^{n} f\right\| \leq(1-a)^{n-1}\|e\|^{\prime}\|e\|\|f\| .
$$

Proof. By Proposition 2.5,

$$
\begin{aligned}
& (n-1) !\left|V^{n} f\right|(t)=\left|\int_{E_{t}}(\phi(t)-\phi(s))^{n-1} f(s) d \mu(s)\right| \\
& \leq(1-a)^{n-1} \cdot \int_{E_{t}}|f(s)| d \mu(s) \leq(1-a)^{n-1}\|e\|^{\prime}\|f\|
\end{aligned}
$$

for almost all $t \in E$. This implies the result.

Letting $a=0$ in Propositions 2.3 and 2.6 gives the following

\section{Corollary 2.7.}

$$
\frac{\left\|\phi^{n}\right\|}{n !\|e\|} \leq\left\|V^{n}\right\| \leq \frac{\|e\|^{\prime}\|e\|}{(n-1) !}
$$

Observe that this Corollary implies that the operator $V$ is quasinilpotent, but not nilpotent.

\section{MAin RESUlts}

Theorem 3.1. For any $a \in[0,1], \quad r_{V}(B(a))=1-a$.

Proof. We use Proposition 2.6 and Corollary 2.7 to obtain

$$
\frac{\left\|V^{n} f\right\|}{\left\|V^{n}\right\|} \leq \frac{n(1-a)^{n-1}\|e\|^{2}\|e\|^{\prime}\|f\|}{\left\|\phi^{n}\right\|}
$$


for every $f \in B(a)$ and every $n \in \mathbb{N}$. Since $\left\|\phi^{n}\right\|^{1 / n} \rightarrow 1$, as $n \rightarrow \infty$ (put $a=0$ in Proposition 2.4) it follows that $r_{V}(f) \leq 1-a$, so that $r_{V}(B(a)) \leq 1-a$. On the other hand, Proposition 2.3 and Corollary 2.7 give

$$
\frac{\left\|V^{n} \chi_{A}\right\|}{\left\|V^{n}\right\|} \geq \frac{\left\|(\phi-a)^{n} \chi_{A}\right\|}{n\|e\|\|e\|^{\prime}}
$$

where $A=\{s \in E: \phi(s) \geq a\}$. Hence $r_{V}\left(\chi_{A}\right) \geq 1-a$ by Proposition 2.4. This completes the proof.

Recall that $L_{V}(a)=\left\{f \in L: r_{V}(f) \leq a\right\}$, where $a \in[0,1]$.

Theorem 3.2. For any $a \in[0,1], \quad L_{V}(a)=\operatorname{Ker} V^{3}+B(1-a)$.

Proof. The proof of the inclusion $L_{V}(a) \subseteq \operatorname{Ker} V^{3}+B(1-a)$ proceeds along the lines of the proof of [1, lemma 4.3] (use Proposition 2.5 and Corollary 2.7 instead of [1, lemma 4.2(a) and (b)]). The opposite inclusion follows by Theorem 3.1 and the obvious fact that $\operatorname{Ker} V^{3} \subseteq L_{V}(0) \subseteq L_{V}(a)$.

The next interesting result now follows easily.

Theorem 3.3. If the operator $V$ is injective, then $L_{V}(a)=B(1-a)$, for all $a \in$ $[0,1]$. Moreover, $\{B(a): a \in[0,1]\}$ is precisely the collection of all quasispectral maximal subspaces of $V$.

Proof. The first statement is clear from Theorem 3.2. Then the identity $L_{V}(a)=$ $B(1-a)$ together with the fact that bands are closed subspaces of $L$ shows that $L_{V}(a)$ is closed, for each $a \in[0,1]$. Hence, $B(a)$ is quasispectral maximal, for each $a \in[0,1]$; see $[1$, theorem 3.1]. If $Y$ is a quasispectral maximal subspace of $V$ and if $a=r_{V}(Y)$, then $r_{V}(B(1-a))=a=r_{V}(Y)$ by Theorem 3.1. This implies that $Y=B(1-a)$, since $Y$ and $B(1-a)$ are both quasispectral maximal subspaces of $V$.

Theorem 3.4. The chain of subspaces $\{B(a): a \in[0,1]\}$ is precisely the family of all bands which are invariant under the operator $V$.

Proof. Let $B$ be a non-trivial band, invariant under $V$. It is not difficult to show that there exists a maximal $a \in[0,1)$ such that $B \subseteq B(a)$. For each $n \in \mathbb{N}$, there exists $f_{n} \in B$ so that $\mu\left(\left\{s \in E: \phi(s) \leq a+1 / n, f_{n}(s) \neq 0\right\}\right)>0$. It follows that there exists $\alpha_{n}>0$ such that the set $C_{n}=\left\{s \in E: \phi(s) \leq a+1 / n,\left|f_{n}(s)\right| \geq \alpha_{n}\right\}$ has positive measure. Since $\left|f_{n}\right| \geq \alpha_{n} \chi_{C_{n}}$, we have $\chi_{C_{n}} \in B$. If $\phi(t) \geq a+1 / n$, then $\left(V \chi_{C_{n}}\right)(t)=\mu\left(C_{n}\right)$ and so $\left(V \chi_{C_{n}}\right) \chi_{A_{n}}=\mu\left(C_{n}\right) \chi_{A_{n}}$ where $A_{n}=\{t \in E$ : $\phi(t) \geq a+1 / n\}$. Since $V \chi_{C_{n}} \in B$ and $B$ is an ideal, it follows that $\chi_{A_{n}} \in B$. If $A=\{t \in E: \phi(t) \geq a\}$, then the identity $\chi_{A}=\sup _{n} \chi_{A_{n}}$ shows that $\chi_{A} \in B$ (as $B$ is a band) and so $B(a) \subseteq B$. Hence, $B=B(a)$.

It is well known (see e.g. [2]) that in the case of the classical Volterra kernel operator $\left(L=L^{2}[0,1], \phi(t)=t\right)$ every invariant closed subspace is equal to $B(a)$ for some $a \in[0,1]$. The following example shows that, in general, $\{B(a): a \in[0,1]\}$ is not even the family of all closed, invariant ideals of $V$.

Example 3.5. Let $L=L^{\infty}[0,1]$, and $\phi(t)=t$. Let $I$ denote the set of all $f \in L$ such that for each $\epsilon>0$ there exists $\delta \in(0,1)$ such that $|f(t)| \leq \epsilon$ for almost all $t \in[0, \delta]$. It is easily seen that $I$ is a closed ideal, invariant under $V$. It is also clear that there is no $a \in[0,1]$ with $I=B(a)$. 


\section{ACKNOWLEDGMENTS}

The author is greatly indebted to Professor Matjaž Omladič for helpful advice. He also wishes to thank the referee for suggestions improving the presentation of the paper.

\section{REFERENCES}

[1] M. Omladič, Quasispectral subspaces of quasinilpotent operators, Proc. Roy. Soc. Edin. 98A (1984), 349 - 354 MR 86f:47004

[2] J. R. Ringrose, Compact non-self-adjoint operators, London: Van Nostrand Reinhold Math. Studies 1971

[3] H. H. Schaefer, Banach lattices and positive operators, (Grundlehren Math. Wiss. Bd. 215) Berlin Heidelberg New York: Springer 1974 MR 54:11023

[4] A. C. Zaanen, Riesz spaces II, Amsterdam: North Holland 1983 MR 86b:46001

Institute of Mathematics, Physics and Mechanics, Jadranska 19, 1000 Ljubljana, SLOVENIA

E-mail address: roman.drnovsek@fmf.uni-lj.si 